\title{
DETERMINATION OF THE ASSIGNED VALUE BY THE PARAMETER OF THE TEST CONTROL SAMPLE USING A COORDINATE MEASURING MACHINE
}

\author{
Abelashvili Nodari, Professor at the Georgian Technical University, Tbilisi, Georgia, \\ ORCID ID: 0000-0003-4002-9177 \\ Didenko Ekaterina, Master at the Georgian Technical University, Process Engineer at Atlantic \\ Georgia LLC, Tbilisi, Georgia
}

DOI: https://doi.org/10.31435/rsglobal_conf/30062021/7621

\begin{abstract}
In this paper will be discussed the theoretical and practical issues of preparing a control sample for geometric parameters of qualification testing using bridge type coordinate measuring machine (CMM) GLOBAL S GREEN 091208.

The use of a control sample prepared in this way ensures the high accuracy of the control parameters of the test item, usually manufactured on the cutting machine (CNC), and the high quality of the determination of measuring instruments, measurement methods and personnel competence.

Theoretical and practical studies have substantiated the possibility of using such a control sample in a machine-building, car-building, aircraft-building and other similar factories.
\end{abstract}

Keywords: qualification testing, inter-laboratory comparison, control sample, Certified value, Assigned value.

1. Introduction. Determining the competence of testing and calibration laboratories is the main purpose of the interlaboratory qualification comparison, the objectivity of which depends significantly on the preparation of a control sample. The accuracy of the production of control sample is not so important as the accuracy of determining the existing linear dimensions of the control geometric parameters, known as the Assigned Value.

2. Purpose and Methods of Research. The process of preparing a control sample for an interlaboratory comparison begins with the creation of a test item and control sample. It is necessary to consider requirements that the control sample should meet. Depending on the model, type, industry and other factors, the test requirements should be adapted to the specific test conditions, taking into account the relevant metrological requirements. The basic requirements that should be met in the control geometry of the inter-laboratory qualification comparison of geometric parameters, include:

- Requirements for control sample surfaces, curvatures, spherical, conical, curved and convex, circular, rectangular, oblong and bevel surfaces;

- Requirements for physical, mechanical, magnetic, chemical and corrosion resistance properties of the control material;

- Requirements for the possibility of making the sample without certain difficulties using standard metal-cutting machine tools;

- Requirements for the universality of the control sample, which means that one control sample should combine the maximum number of control points or surfaces listed above;

- The design and construction requirements should ensure the ergonomics of the control sample, and its dimensions should meet the limits of the measurement area of a particular measuring machine in the direction of the $\mathrm{X}, \mathrm{Y}, \mathrm{Z}$ axes;

- No special measurement requirements are needed for the accuracy of production of the control sample (surfaces, grooves, etc.), as the aim of the inter-laboratory comparison is to trace the real size with maximum accuracy and reliability;

- It is important that the control sample maintained the stability of geometric parameters, both in operation and transportation, as well as in the influence of environmental factors, such as temperature, humidity, dust, grease, mechanical damage, vibration, impact and other factors;

Given the above, a control sample of complex geometric shape was prepared (Fig. 1), aluminum was selected as the material based on physical, chemical, mechanical properties and requirements of the qualification testing. 


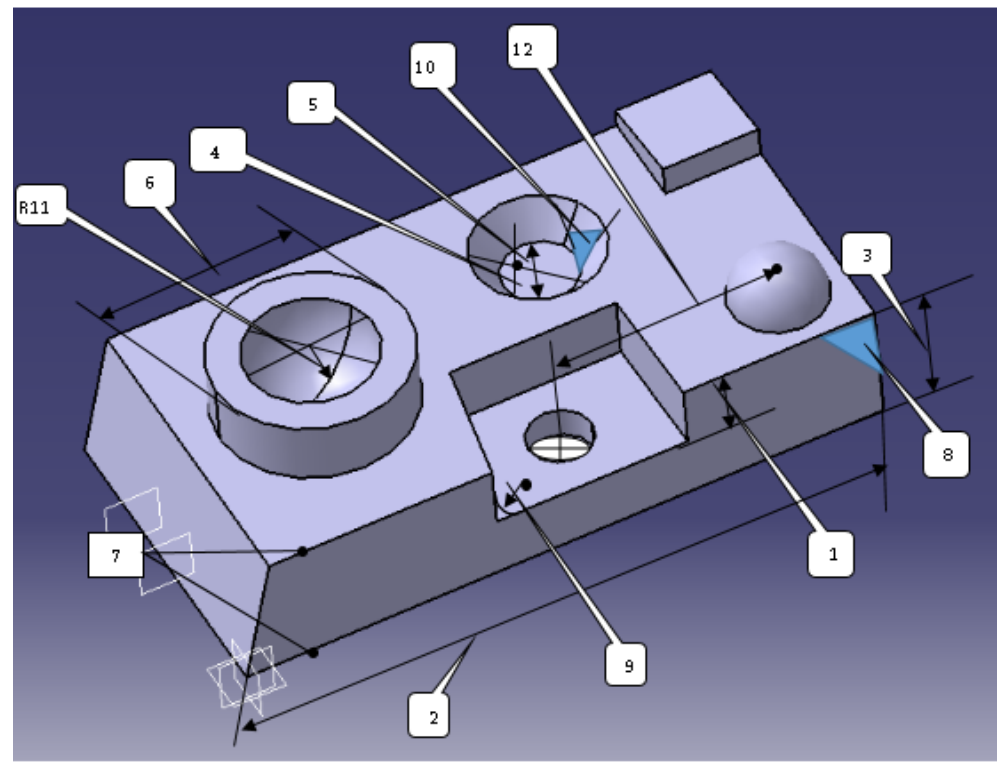

Fig. 1. The control sample for qualification testing of geometric parameters

In the table below are shown possible test parameters of the control sample which can become objects of measurement during the approbation and qualification testing (Table 1).

Table 1. Control sample study zones

\begin{tabular}{|l|l|}
\hline Position on the drawing & Test parameter \\
\hline $1,2,3$ & Linear dimensions \\
\hline 4 & Depth \\
\hline 5 & Inner diameter \\
\hline 6 & Outer diameter \\
\hline 7 & Parallelity of curves and planes \\
\hline 8 & Perpendicularity of ridges and planes \\
\hline 9 & Radius \\
\hline 10 & Angle \\
\hline 11 & The inner radius of the sphere \\
\hline 12 & Distance between centers \\
\hline
\end{tabular}

After production of the control sample, GLOBAL S GREEN 091208 bridge type coordinate measuring machine $(\mathrm{CMM})$ approves measurements of the control points, and determines Assigned Values of the control parameters. To ensure this, we use the requirements of ISO 13528-2015 standard (Section 7.5) according to which: "An assigned value can be determined by a single laboratory using a reference method, such as, for example, a primary method. The reference method used should be completely described and understood, and with a complete uncertainty statement and documented metrological traceability that is appropriate for the proficiency testing scheme. The reference method should be commutable for all measurement methods used by participants." [1]. It (Section 7.5.2.) also states that "assigned value of the proficiency test item can be derived by a single laboratory using a suitable measurement method, from a calibration against the reference values of a closely matched certified reference material."

This approach assumes that data of certified (CRM) value is compatible with all measurement methods used by participating laboratories, requiring a series of measurements to be performed on a control sample using the same method of measurement under reproducibility.

In this case, to calculate the Assigned Value of the qualification testing, we can apply the following formula

$$
x_{p t}=x_{C R M}+\bar{d}
$$

where: $x_{C R M}$ - is the assigned value for the CRM;

$x_{p t}$ - is the assigned value for the proficiency test item; 
the $i^{\text {th }}$ samples;

$d_{i}$ - is the difference between the average results for the proficiency test item and the CRM on

$\bar{d}$ - is the average of the differences $d_{i}$.

The control sample with non-precision geometrical dimensions was made with respect to above mentioned requirements. Sample was manufactured from aviation grade aluminum on the CNC machine (Fig. 2).

For the approbation of the control sample measurements was chosen the distance between the Lowest Point- 1 of a concave cylinder and the Highest Point- 2 of the convex sphere. Here we have the values of the DIST_LOW-HIGH coordinates.

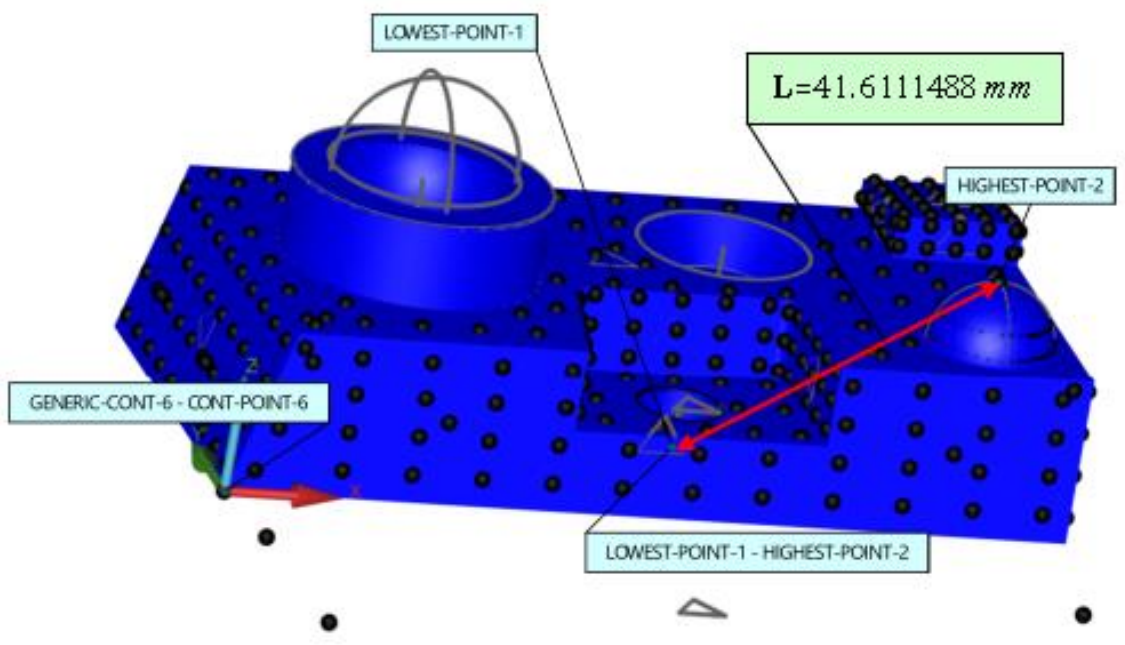

Fig. 2. Control sample to be measured at points 1, 2 and 6

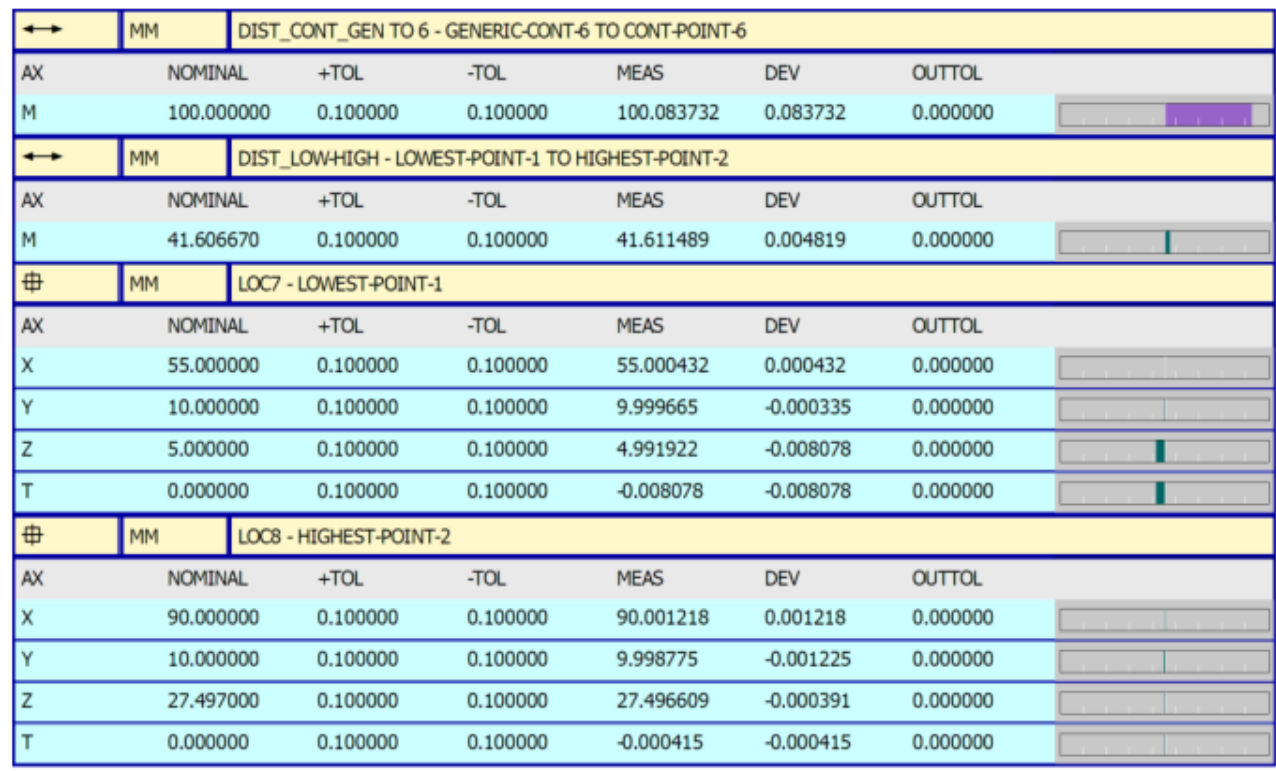

Fig. 3. Measuring machine test protocol

The result of the approbation performed on the control sample for inter-laboratory comparison of geometric parameters by CMM machine is shown in Fig 3.

\section{Research results}

In the report we see the nominal values of the $\mathrm{X}, \mathrm{Y}, \mathrm{Z}$ coordinates of the indicated points and for each of them a tolerance value of $\pm 0,1 \mathrm{~mm}$ is indicated. (Fig 3.)

The spatial coordinates of point 1 and point 2 are also defined, limits of the tolerance interval are indicated for each, and spatial coordinates are given which indicate the deviation values. The report also indicates the measured distance between points 1 and 2 which is $41,611489 \mathrm{~mm}$. 
Based on the coordinate data of the CMM machine, it is possible to calculate the result algebraically.

For these points $(1,2)$, according to the coordinates, we can calculate the distance between them using well-known formula

$$
\begin{gathered}
d=\sqrt{\left(x_{2}-x_{1}\right)^{2}+\left(y_{2}-y_{1}\right)^{2}+\left(z_{2}-z_{1}\right)^{2}}= \\
\sqrt{(90,001218-55,000432)^{2}+(9,998775-9,999665)^{2}+(27,496609-4,991922)^{2}}= \\
\sqrt{1225,055+0,0000007921+506,460936}=\sqrt{1731,515934}=\mathbf{4 1 , 6 1 1 4 8 8}(\mathbf{m m})
\end{gathered}
$$

Algebraically calculated distance value with a precision of $1 \mu \mathrm{m}$ was actually matched with the value measured by the coordinate measuring machine, indicating the full adequacy of the theoretical and practical results.

In the same way, we can calculate the deviation values defined in the report, which literally represent the Type A uncertainty.

$$
\begin{gathered}
d e v_{1-2}=\sqrt{\left(d e v_{x 1}+d e v_{x 2}\right)^{2}+\left(d e v_{y 1}+d e v_{y 2}\right)^{2}+\left(d e v_{z 1}+d e v_{z 2}\right)^{2}} \\
=\sqrt{0.0000027+0.000021+0.000072}=0.00978 \approx 0.001 \mathrm{~mm}
\end{gathered}
$$

In such case in the formula (1) given for the calculation of the Assigned Value to the qualification testing, (1) $x_{C R M}$ - represents $d$ lengths of the search section and $\bar{d}$ - deviation with respect to each axis. Therefore formula (1) will be

$$
x_{p t}=d+d e v_{1-2}
$$

Based on the above mentioned formula distance between the Lowest Point and the Highest Point will be:

$$
x_{p t}=L=41,611488 \pm 0.001[\mathrm{~mm}]
$$

which is the Assigned Value of the given parameter of control sample.

4. Conclusions. Based on the theoretical analysis and practical research, it was determined that the high-precision bridge type coordinate measuring machine can be used for qualification testing of geometric parameters, to determine the control parameters of the control sample with high accuracy and Assign Values to these parameters.

The requirements of ISO 13528-2015 standard have been met to determine the value given to a specific bridge type coordinate measuring machine (type GLOBAL S GREEN 091208, serial number GLCS001303IA), which has undergone the calibration procedure (Calibration Certificate No: SC20002771) with components according to the ISO 10360-2 (2001) methodology, meets the metrological traceability requirements according to which "The calibration equipment calibrated by accredited laboratories and are traceable to the national and international reference standards".

\section{REFERENCES}

1. ISO 13528:2015. Statistical methods for use in proficiency testing by interlaboratory comparison. p.97. Retrieved from https://www.iso.org/standard/56125.html

2. N.N. Abelashvili ${ }^{1}$, N.N. Abelashvili ${ }^{2}$ Treatment Results in Laboratory Trials Environment LabVIEW. Collection of Proceedings XV international conference NIDays 2016 Moscow, November 25, 2016. 